\title{
Spatially resolving the accretion shocks on the rapidly-rotating MO T-Tauri star MN Lupi ${ }^{\star}$
}

\author{
K. G. Strassmeier ${ }^{1}$, J. B. Rice ${ }^{2}$, A. Ritter ${ }^{1}$, M. Küker ${ }^{1}$, G. A. J. Hussain ${ }^{3, \star \star}$, S. Hubrig ${ }^{4}$, and R. Shobbrook ${ }^{5}$ \\ 1 Astrophysical Institute Potsdam, An der Sternwarte 16, 14482 Potsdam, Germany \\ e-mail: [KStrassmeier;ARitter; MKueker]@aip.de \\ 2 Department of Physics, Brandon University, Brandon, Manitoba R7A 6A9, Canada \\ 3 Harvard Smithsonian Center for Astrophysics, Cambridge, USA \\ ${ }^{4}$ European Southern Observatory, 19001 Santiago, Chile \\ 5 Research School of Astronomy and Astrophysics, Australian National University, Cotter Road, Weston, ACT 2611 Australia
}

Received 18 February 2005 / Accepted 9 June 2005

\begin{abstract}
We obtained high-resolution, high-quality VLT/UVES spectra to reconstruct the two-dimensional surface structure of the rapidly-rotating classical T-Tauri star MN Lupi on two separate nights. Both surface maps show a structured warm $(5000 \mathrm{~K})$ band centered around the pole at a latitude of $\approx 65^{\circ}$. Located within the band are two hot spots with temperatures of approximately or possibly even in excess of $5800 \mathrm{~K}$, i.e. $2000 \mathrm{~K}$ above the effective photospheric temperature. Both maps appear with an adjacent equatorial band of temperature $3400 \mathrm{~K}$, some $400-500 \mathrm{~K}$ below the effective photospheric temperature. While we interpret the two hot spots and the warm high-latitude band to be the heating points from two accretion impacts at the time of our observations and their redistributed energy trailed due to the fast stellar rotation, respectively, the cool equatorial band may not be cool after all but due to obscuration of the stellar surface by the innermost region of the disk. The fact that the hot spots appear at high stellar latitude is in agreement with the magnetospheric accretion model that proposes material funnelling onto the star along a predominantly dipolar magnetic field at roughly $50^{\circ}$ latitude. The evidence of ongoing disk accretion, together with the very fast rotation of MN Lupi of just 3-4 times below its break-up velocity, suggests that the accretion mechanism is the cause of its rapid surface rotation. We present a model of magnetic star-disk coupling for MN Lupi that predicts a polar surface magnetic field of $\approx 3 \mathrm{kG}$.
\end{abstract}

Key words. stars: activity - starspots - stars: imaging - stars: individual: MN Lup - stars: individual: T Tauri stars: pre-main sequence

\section{Introduction}

MN Lupi = RX J1523.5-3821 (RA 1523 30.2, Dec -3821 26 (2000.0, FK5), $V=14.3, \mathrm{~m} 0$ ) is a very young star in the Lupus star-forming region and was discovered on the basis of its ROSAT X-ray emission (Krautter et al. 1997). It was listed as a weak-lined T Tauri star (WTTS) in subsequent studies of the Lupus region. Wichmann et al. (1998a) discovered its photometric variability due to starspots with a rotation period of 0.44 days and obtained first estimates of its age ( $\approx 2 \mathrm{Myr})$ and mass $\left(0.30 M_{\odot}\right)$.

At a distance of just $150 \mathrm{pc}$, the Lupus region is one of the nearest star-forming regions. The bulk of (known) stellar components are in the age range between a few $10^{6} \mathrm{yr}$ and $10^{8} \mathrm{yr}$, i.e., the age between the oldest $T$ Tauris and the youngest ZAMS dwarfs (Wichmann et al. 1998a). Again based on the Krautter et al. sample, Wichmann et al. (1997) found that the

* Based on observations collected at the European Southern Observatory, Paranal, Chile.

$\star \star$ Now at ESA/ESTEC, Nordwijk, The Netherlands. mean age of weak-line $\mathrm{T}$ Tauri stars seen in projection against dark clouds is lower than the mean age of WTTSs far away from them, but still higher than the mean age of the cluster's classical T Tauri stars (CTTS). This makes the Lupus region an interesting laboratory for studies of infant evolutionary processes and their interrelations.

One of the questions to be answered for CTTSs in general is the relation between accretion disk parameters, especially the accretion rate, and the evolution of the central star, and how does it all tie into the basic magnetospheric model and its numerous variants. CTTSs are believed to have an accretion disk with a number of complex physical processes that cause, among other effects, an excess of radiation in the IR and the UV due to reradiation off the disk and the appearance of accretion shocks in the stellar atmosphere, respectively (e.g. Hartmann et al. 1994). CTTSs are not necessarily always younger than WTTSs because a disk's size is largely determined by the initial conditions of star formation rather than subsequent evolution (Bertout 1989). CTTS optical spectra are dominated by very strong emission lines, usually 
hydrogen and forbidden oxygen lines but also resonance lines such as Ca II H and K. The WTTSs, on the other hand, are thought to be without a disk, i.e. "naked" (cf. Walter 1987), and thus without the large IR and UV excess. Particularly the photospheric lithium abundance may be a good WTTS indicator. Many of them still show signs of circumstellar material but largely without accretion. However, some of our previous Doppler images of WTTSs showed occasionally hot spots (e.g. Strassmeier \& Rice 2000; Rice \& Strassmeier 1996) while others did not (Strassmeier \& Rice 1998). Also, no veiling of the photospheric spectrum was apparent in these stars. Finally, most of the ZAMS dwarfs show a solar-like optical/UV spectrum without the aforementioned irregularities and with almost all the surface lithium depleted. A good overview of previous results from high-resolution spectroscopy of CTTSs was given in the discussion by Stempels \& Piskunov (2002), and we refer the reader to this paper for further references.

Our approach in the present and forthcoming paper is to analyze phase-resolved, high-resolution and high signal-to-noise optical spectra of selected targets that may be in an evolutionary transition phase, either from CTTS to WTTS or from WTTS to ZAMS. Our direct goal is to disentangle the spectral signatures of the accretion flow from the chromospheric and photospheric magnetic activity by spatially resolving the stellar surface with the technique of Doppler imaging. From these maps we hope to be able to, among other objectives, constrain the accretion rates and whether or not these are consistent with the general magnetospheric model of disk accretion at the evolutionary state of the central star under investigation. Mapping a series of suitable stars of various ages may then lead to quantitative values to be compared to model predictions of the magnetospheric star-disk interaction, in particular those of Küker et al. (2003).

Section 2 describes our observations and data reductions. Astrophysical parameters of MN Lupi are determined in Sect. 3, the results from the Doppler-imaging analysis are described in Sect. 4. Section 5 is an attempt to model the observed surface structure with a magnetospheric star-disk model. Section 6 summarizes our conclusions.

\section{Observations and data reduction}

\subsection{Previous observations of MN Lupi}

The $\mathrm{H} \alpha$ emission and the strong lithium absorption were already discovered from a $R \approx 1500$ spectrum obtained by Krautter et al. (1997) in 1992/93. We are aware of only one further, $R \approx 20000$ ESO-CASPEC spectrum in the range 580-850 $\mathrm{nm}$ obtained by Wichmann et al. (1999) in 1995. According to this spectrum, MN Lupi would be a double-lined spectroscopic binary with radial velocities of -12.6 and $+23.8 \mathrm{~km} \mathrm{~s}^{-1}$ and projected rotational velocities of 50-55 $\mathrm{km} \mathrm{s}^{-1}$ and $27 \mathrm{~km} \mathrm{~s}^{-1}$ for the two components, respectively (Table 1 in Wichmann et al. 1999). H $\alpha$ was already interpreted to be multiple based on the older $R \approx 1500 \mathrm{spec}-$ trum that showed a profile asymmetry in the red wing (Fig. 5 in Krautter et al. 1997). In the present paper, we can not verify the spectroscopic binary nature of MN Lupi nor do we see a persistent $\mathrm{H} \alpha$-line asymmetry. We attribute this to the low resolution and the comparably low quality of the previous data.

\subsection{New VLT observations}

High-resolution spectroscopic observations were obtained with the Ultraviolet Visual Echelle Spectrograph (UVES, Dekker et al. 2000) at the 8.2-m Kuyen telescope of the VLT in Chile during two consecutive nights on May 26/27 and 27/28, 2000. UVES's blue and red arm were used simultaneously and gave a nearly continuous wavelength coverage from $340 \mathrm{~nm}$ to $680 \mathrm{~nm}$ with a spectral resolution of $R \approx 60000$ (there is a small gap from $450-478 \mathrm{~nm}$ due to the dichroid). The three-slice BowenWalraven image slicer \#1 was used, which projects the $2.1^{\prime \prime} \times$ $2.6^{\prime \prime}$ entrance aperture to a $0.68^{\prime \prime} \times 7.9^{\prime \prime}$ slit. The CCD system of UVES consists of a blue-sensitive EEV 44-82 $2 \mathrm{k} \times 4 \mathrm{k}$ device with a measured QE of $55 \%$ at $350 \mathrm{~nm}$ and a mosaic of a red-optimized EEV $2 \mathrm{k} \times 4 \mathrm{k}$ version and a red-sensitive MIT CCID-20 $2 \mathrm{k} \times 4 \mathrm{k}$ device with a measured QE of $80 \%$ at $800 \mathrm{~nm}$. A gap between the two chips of width $0.96 \mathrm{~mm}$, or $6415-\mu \mathrm{m}$ pixels, causes an additional gap in the spectral coverage of $\approx 10 \mathrm{~nm}$ from $574 \mathrm{~nm}$ to $584 \mathrm{~nm}$.

Seeing was always between $0.6-1.0^{\prime \prime}$ and all red integrations were set to an exposure time of $2100 \mathrm{~s}$. This allowed for a total of 17 spectra of MN Lupi in the first night and 16 spectra in the second night. The series also included 8 uv/blue integrations per night (with twice the integration time). A few of these spectra suffered from large amounts of cosmic-ray hits due to their low signal-to-noise $(\mathrm{S} / \mathrm{N})$ ratio. The red-wavelength spectra have $\mathrm{S} / \mathrm{N}$ ratios of typically 100:1 per single exposure per pixel while the uv/blue spectra range from a minimum of $\approx 3: 1$ between $320-340 \mathrm{~nm}$ (not shown in Fig. 1) to a maximum of $30: 1$ at $420 \mathrm{~nm}$.

Stellar comparison targets were also observed with the same set-up, among them the active single M-dwarfs HK Aqr (=Gliese 890, M 1.5), EQ Peg (M 3.5), HD 119850 (M 1.5) and the comparably inactive stars HD 131976 (M 1), HD 196795 (K5V) and HD 209290 (M0). A single $R=$ 120000 spectrum of the bright M0V MK-standard HD 202560 (AX Mic) centered at our typical Doppler-imaging wavelength $\lambda 642 \mathrm{~nm}$ was obtained at CFHT with the Gecko spectrograph on Aug. 27, 2004 and used for a visual comparison. Table 1 gives an overview of the VLT observing log.

Figure 1 displays a UVES spectrum of MN Lupi from $360 \mathrm{~nm}$ to $680 \mathrm{~nm}$ along with the inactive M 0 comparison star HD 209920.

\subsection{VLT/UVES data reduction}

Calibration frames included Th-Ar comparison-lamp spectra, several series of flat-field spectra from a quartz lamp, and numerous CCD biases.

The echelle spectra were reduced and extracted using the Image Reduction and Analysis Facility (IRAF). The bad-pixel correction, bias subtraction, flat fielding and cosmic-rays detection was done using the ccdred package. Bias levels were removed by subtracting the median overscan from each image, 


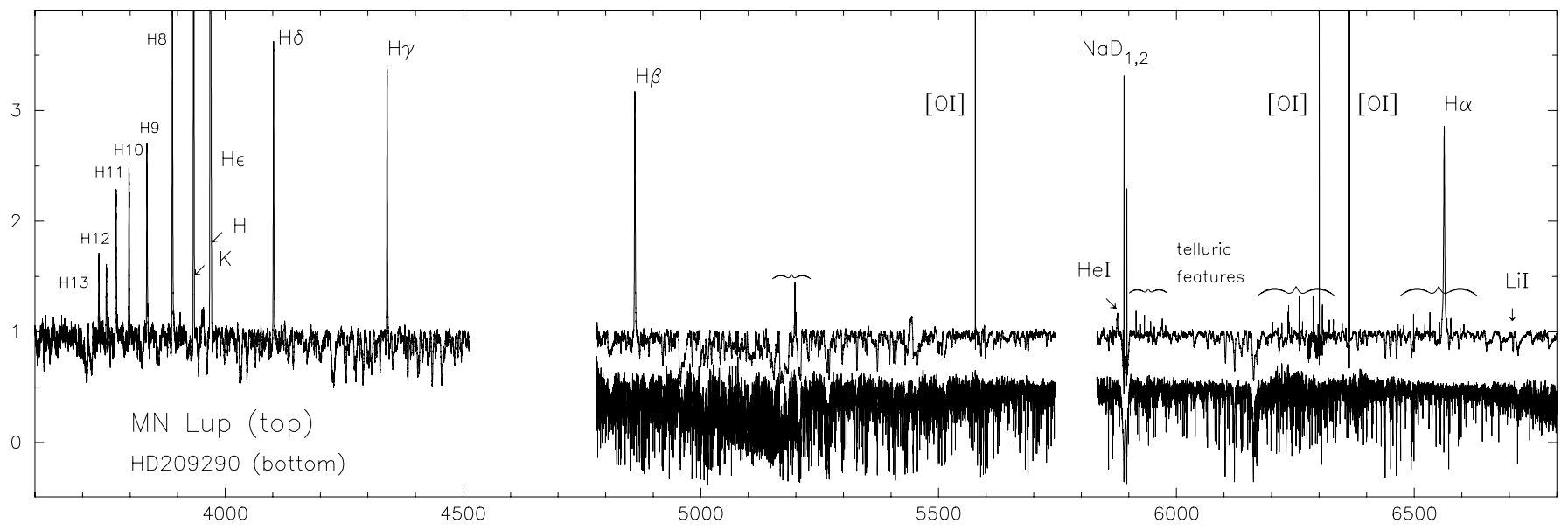

Fig. 1. Optical and UV spectrum of MN Lupi (top spectrum) in comparison with the inactive M0-star HD 209290 (bottom spectrum, shifted by 0.5 in intensity). All hydrogen Balmer lines up to the Balmer jump appear in emission. Strong Ca II $\mathrm{H}$ and $\mathrm{K}$, Na D, and $\mathrm{He}$ I emission are evident as well as numerous weak telluric features. The very strong forbidden oxygen O I lines are geocoronal in origin. Also notice the lithium absorption line at $670.8 \mathrm{~nm}$.

Table 1. VLT/UVES observing log. Listed is the wavelength range $\lambda$, number of spectra $N$, their exposure time $t$ in seconds, and the averaged heliocentric radial velocity $v_{\mathrm{r}}$. Values for MN Lupi are from this paper (errors are rms).

\begin{tabular}{llllll}
\hline \hline $\begin{array}{l}\text { Date } \\
\text { UT2000 }\end{array}$ & Object & $\begin{array}{l}\lambda \\
(\mathrm{nm})\end{array}$ & $\begin{array}{l}t \\
(\mathrm{~s})\end{array}$ & $\begin{array}{l}v_{\mathrm{r}} \\
\left(\mathrm{km} \mathrm{s}^{-1}\right)\end{array}$ \\
\hline May 27 & MN Lup & $320-450$ & 8 & 4200 & $\ldots$ \\
& MN Lup & $480-680$ & 17 & 2100 & $4.7 \pm 1.0$ \\
& Sun & $480-680$ & 1 & 600 & 0.0 \\
& HD 119850 & $320-450$ & 1 & 180 & $\ldots$ \\
& HD 119850 & $480-680$ & 1 & 180 & 15.809 \\
& HD 196795 & $480-680$ & 1 & 180 & -40.9 \\
& HD 209290 & $480-680$ & 1 & 180 & +18.363 \\
May 28 & MN Lup & $320-450$ & 8 & 4200 & $\ldots$ \\
& MN Lup & $480-680$ & 16 & 2100 & $4.8 \pm 2.9$ \\
& Sun & $480-680$ & 1 & 600 & -0.022 \\
& EQ Peg & $320-450$ & 1 & 200 & $\ldots$ \\
& EQ Peg & $480-680$ & 1 & 200 & $\ldots$ \\
& HK Aqr & $320-450$ & 1 & 180 & $\ldots$ \\
& HK Aqr & $480-680$ & 1 & 180 & +6.60 \\
& HD 131976 & $320-450$ & 1 & 120 & $\ldots$ \\
& HD 131976 & $480-680$ & 1 & 120 & +27.9 \\
\hline
\end{tabular}

followed by the subtraction of a median (already overscan subtracted) bias frame. The images were flattened by dividing by a median, normalized flat. After the removal of cosmic rays the one-dimensional spectra were extracted, wavelength calibrated and normalized using the echelle package. The extraction of the one-dimensional spectra was done from summed apertures of between $42-46$ pixels width centered on the spectra. No profile weighting was presumed. The target spectra were then wavelength calibrated using the consecutively recorded Th-Ar arc spectra. No background sky-subtraction could be performed due to the use of an image slicer. Therefore, telluric emission features were not removed during the reduction process (neither were absorption features). Finally, the extracted spectra were continuum normalized. Notice in Fig. 1 that the continuum around the Balmer jump at $370 \mathrm{~nm}$ was set such that the spectrum appears flat on both sides of the jump. No absolute flux calibration was attempted.

\subsection{Photometry}

Follow-up photometry of MN Lupi was obtained at Siding Spring Observatory using the Australian National University's $61-\mathrm{cm}$ reflector on 8 nights between June 4-12, 2002. The telescope was equipped with a thermoelectrically cooled RCA 31034A photomultiplier photometer and filters that match the Johnson BVRI system. The filters are as listed by Bessell (1979, 1990). A 30" aperture was used. The $B$ band measurements are the noisiest as they had the smallest integration times (16 s; 1300 counts) but they were obtained just to get an independent average $B-V$ value. The other bandpasses all had $80 \mathrm{~s}$ integration time and count rates of approximately $30 \mathrm{k}$, $70 \mathrm{k}$ and $100 \mathrm{k}$ for $V, R$, and $I$, respectively. All magnitudes are relative to the comparison star HD 136736 (K2III). Its parameters are found to be: $V=9.296 \pm 0.020, B-V=1.26$, $V-R=0.67$, and $V-I=1.28$. Errors of the colors are approximately \pm 0.03 . The constancy of the comparison star was checked against a third star, HD 136647, and proofed to be constant to within 0.02 over the course of the eight nights. Its parameters are found to be: $V=9^{\mathrm{m}} \cdot 628 \pm 0.020, B-V=0$. 29 , $V-R=0.15$, and $V-I=0.31$. These values were determined from one night on which four standards were observed. Being much brighter than MN Lupi, both comparison stars were given only one-half of the total integration time of the variable and were measured to a higher internal precision of between 0.005 and $0 . \mathrm{m} 010$. 


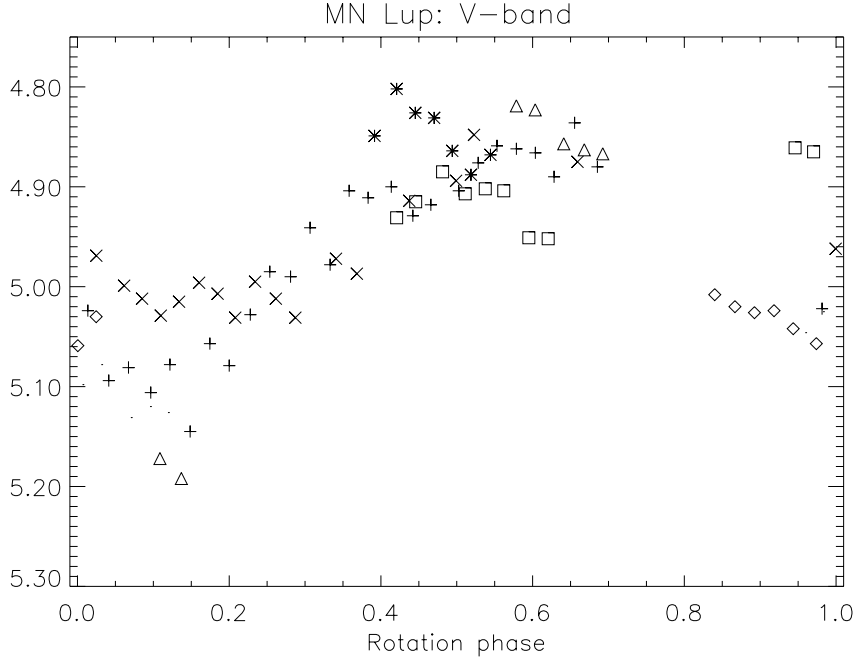

Fig. 2. Differential $B V R I$-photometry of MN Lupi phased with $P=$ 0.439 days (only $V$ is shown). Different symbols refer to different nights, i.e. JD $2452400+$ (night number) as following: plusses $=+30$, asterisks $=+31$, diamonds $=+33$, triangles $=+34$, squares $=+35$, dots $=+36$, crosses $=+37$.

\section{MN Lupi = RX J1523.5-3821}

\subsection{Photometric variations}

The light variability of MN Lupi was discovered by Wichmann et al. (1998a) as part of their survey of T Tauri stars in the Lupus star forming region. Our new data from June 2002 confirm the variability of MN Lupi and show a brightness variation of $\approx 0$. 1 from night to night (Fig. 2). Ignoring a couple of very spurious points likely due to clouds, we find the following "least-spotted" photometric values from the peak in the light and color curves: $V=14.13 \pm 0.03, B-V=1 \mathrm{~m} .46 \pm 0.05$, $V-R=1 \mathrm{~m} .05 \pm 0.03$, and $V-I=2 \mathrm{~m} 33 \pm 0.05$. Without extinction this would correspond roughly to an effective surface temperature of $4070 \mathrm{~K}$ according to the $B-V$ calibration of Flower (1996) but more likely to $3800 \mathrm{~K}$ from a comparison with model atmospheres from Bessell et al. (1998) and Kurucz (1993). We note though that our unspotted magnitudes likely underestimate the true unspotted magnitudes because a certain spottedness likely remained even during light-curve maximum. The $B-V$ vs. $V-I_{\mathrm{C}}$ calibration from the Hipparcos-Tycho sample (see Strassmeier \& Schordan 2000) suggests a $V-I_{\mathrm{C}}$ of 1.5 for MN Lupi, i.e. $1^{\mathrm{m}} 95$ in the Johnson system according to Bessell (1979). To estimate interstellar extinction from broad-band photometry, Gullbring et al. (1998) suggested to use the $V-R$ color excess rather than $B-V$ or $V-I$ because veiling, if present, does not change significantly between the $V$ and the $R$ wavelengths (see also Hartigan et al. 1995). MN Lupi's M 0 classification in Table 2 is from a direct comparison with the spectra of our reference stars (see Fig. 1) and suggests an intrinsic $V-R_{0}=1.28$ according to the table in Cox (2000). Therefore, $A_{\mathrm{V}}=0.74$ for MN Lupi (or $A_{\mathrm{V}}=-0.2$ from $B-V$ ). The $B-V$ of 1.46 and the $V-I$ of 2.33 indicate a weak $U$ and IR excess, respectively. Keeping in mind that some additional uncertainties arise when matching a normal $\mathrm{M}$ dwarf to a

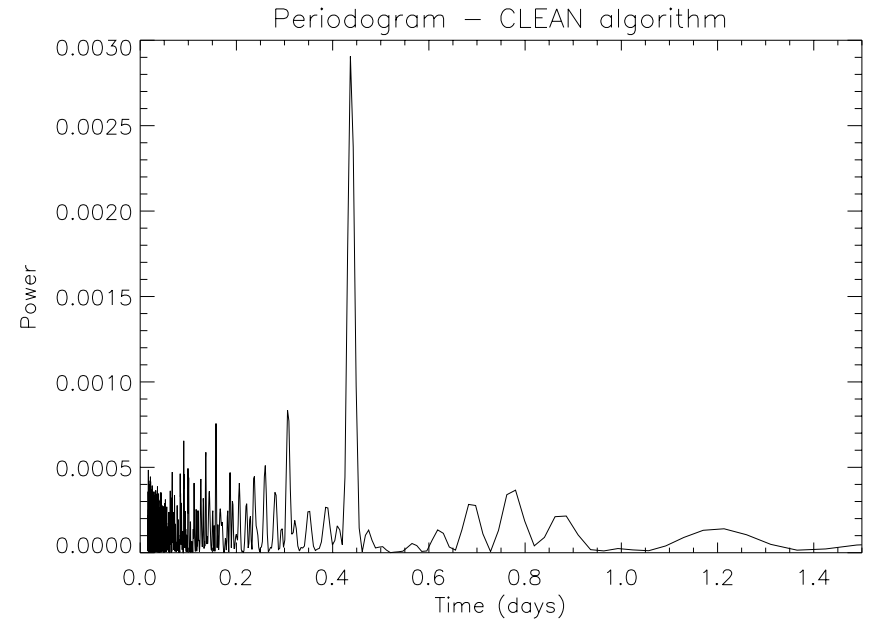

Fig. 3. Periodogram of MN Lupi from the CLEAN algorithm. The peak at 0.439 days is interpreted to be the rotational period.

T Tauri M-star spectrum, we conclude that MN Lupi is indeed a M 0 pre-main-sequence star.

\subsection{Rotational period}

Wichmann et al. (1998a) had already found a period of 0.44 days but deemed it very uncertain given their sparse data and the small amplitude. We applied two different periodsearch algorithms to our new data, a modified Lomb-Scargle method and the CLEAN approach - the latter deconvolves the spectral window from the Fourier power spectrum. The periodogram from the CLEAN algorithm is shown in Fig. 3 and shows a single well-defined peak at $0.439 \pm 0.005$ days, in excellent agreement with Wichmann's 0.44-day value. The Lomb-Scargle periodogram also shows the 0.44-day period as the strongest peak but additionally a comparable but formally weaker peak at 0.31 days, and an even weaker one at 0.78 days.

We interpret the 0.44 -day period to be the rotation period of MN Lupi and phase all data in this paper according to the following ephemeris

$\mathrm{HJD}=2451691.478825+0.439 \times E$,

where the zero point is the time of the first spectrum of our time series and is an arbitrary time.

\subsection{Radial velocity variations}

Figure 4 shows our photospheric velocity measurements during the two consecutive nights of observation. Despite having error bars that are relatively large due to the broad and variable spectral lines of MN Lupi, we detect a peak-to-peak velocity variations of $\approx 9 \mathrm{~km} \mathrm{~s}^{-1}$ during the second night and $\approx 3 \mathrm{~km} \mathrm{~s}^{-1}$ during the first night. Velocities were determined by cross correlating all combined echelle orders from one CCD with our comparison stars (listed in Table 1). Regions with emission lines were excluded. Firstly, we cross correlated the reference stars within each other to verify their adopted values. As primary standard, the Sun (a twilight sky spectrum) and HD $209290\left(+18.363 \mathrm{~km} \mathrm{~s}^{-1}\right.$, Nidever et al. 2002) were 


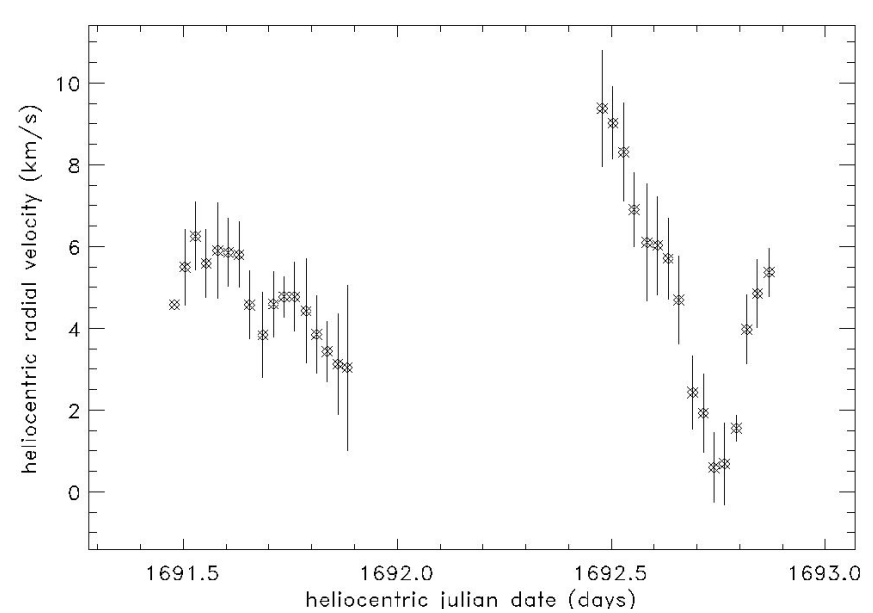

Fig. 4. Heliocentric radial velocities of MN Lupi for both nights. Only absorption lines were used for the cross correlation in order to best represent the photospheric velocities. Note that the error bars are rms values from individual measures of many different wavelength regions relative to the first spectrum and represent best estimates of the true external errors.

adopted. Secondly, all echelle orders for MN Lupi were cross correlated with HD 209290 as the zero point. This gave comparably large error bars because of the large width of the Gaussian fit function and the variations of the line profiles. Therefore, we preferred to cross correlate all single wavelength regions without emission lines one by one with the appropriate wavelength region of the first MN Lupi image as a reference. Then the mean and rms of these results are built, followed by a comparison of the resulting mean value to the mean of the heliocentric velocities from the cross correlation with HD 209290. The rms of an individual measure in Fig. 4 is $\approx 0.7 \mathrm{~km} \mathrm{~s}^{-1}$.

The two-night average heliocentric velocity of MN Lupi is $4.77 \pm 2.0$ (rms) $\mathrm{km} \mathrm{s}^{-1}$, with a peak-to-peak range from $0.5-9.5 \mathrm{~km} \mathrm{~s}^{-1}$. This compares to the $0 \mathrm{~km} \mathrm{~s}^{-1}$ of the core clouds of Lupus from CO-band observations (Dame 1987) and with the $-0.03 \pm 1.2 \mathrm{~km} \mathrm{~s}^{-1}$ of the combined CTTS sample of Dubath et al. (1996) and Wichmann et al. (1999), and the $1.3-3.2 \mathrm{~km} \mathrm{~s}^{-1}$ from the ROSAT WTTSs in Lupus (Wichmann et al. 1999). MN Lupi clearly belongs to the Lupus star-forming region but its position and velocity suggests it lies away from the central CO clouds.

\subsection{The emission-line spectrum}

The observed optical spectrum is dominated by hydrogen and forbidden oxygen lines but also by the resonance lines of $\mathrm{Ca}$ II $\mathrm{H}$ and $\mathrm{K}$ and $\mathrm{Na} \mathrm{I}_{1,2}$, and a bulk of comparably weak telluric features, the so-called airglow emission lines (Fig. 1). Hanuschik (2003) presented a high-resolution atlas of optical sky emission lines as measured with UVES, which includes the strong forbidden oxygen lines. Obviously, a number of different formation regions and mechanisms cause the emission-line spectrum of MN Lupi.

Balmer emission is present all the way down to the Balmer jump. Visual inspection indicates emission up to at least H13, with the $\mathrm{H} 8$ emission being the strongest of all Balmer lines.
Balmer lines
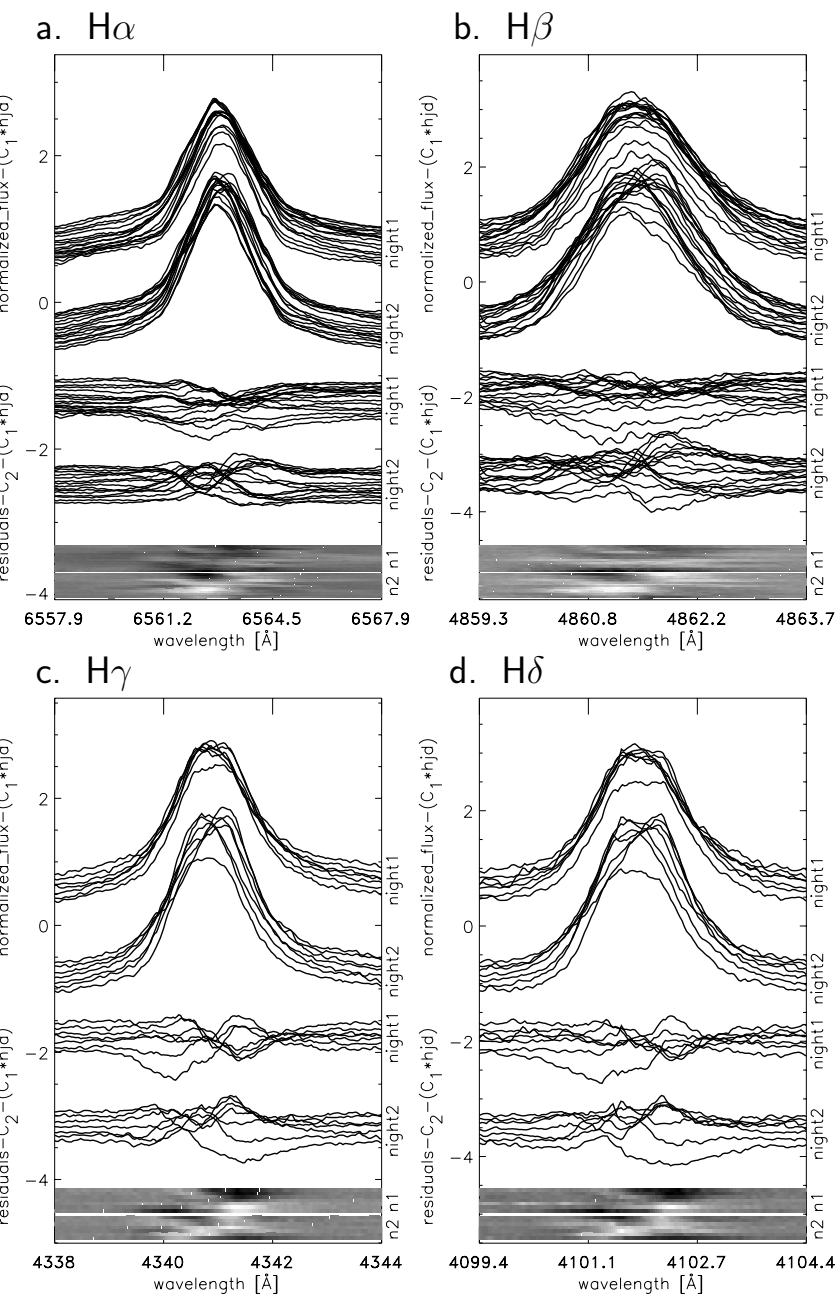

Fig. 5. Evolution of the observed Balmer line profiles. Each panel shows the original spectra in the top, the residuals after subtraction of an average spectrum in the middle, and a grey scale plot of the residuals in the bottom. Time runs from top to bottom with individual spectra offset by an arbitrary constant $C . n 1$ refers to the first night, $n 2$ to the second night. Note the dark and bright features that consistently appear throughout the emission profiles. The average equivalent widths are $-4.7 \AA( \pm 0.8),-3.9 \AA( \pm 0.9),-3.45 \AA( \pm 0.45)$, and $-4.0 \AA$ $( \pm 0.6)$ for $\mathrm{H} \alpha, \mathrm{H} \beta, \mathrm{H} \gamma$, and $\mathrm{H} \delta$, respectively. The number in parentheses is the peak-to-peak variation.

$\mathrm{S} / \mathrm{N}$ ratio for the UV part of our blue-wavelength spectra is between 10-15:1. Figure 5 compares time series of the line profiles of $\mathrm{H} \alpha$ through $\mathrm{H} \delta$ and indicates their consistent changes. The average width of the $\mathrm{H} \gamma$ core-emission component is $\approx 270 \mathrm{~km} \mathrm{~s}^{-1}$ with a total equivalent width of $-4.1 \pm$ 0.4 (rms) A. There is a similar value for the other lines, indicating that these lines form far above the stellar photosphere, likely in the freely falling gas channelled along magnetic field lines outside of the accretion shock (see Sect. 5). The $\mathrm{H} \alpha$ origin is clearly more complex, as indicated by its non-Gaussian shape and large equivalent width of $-6.73 \pm 0.52$ (rms) $\AA$. The high-excitation $\mathrm{H}$ lines (H8-H13) seem to originate closer to or even within the accretion shock(s) near the stellar surface that should also give rise to a hot continuum excess. We will use 


\section{Accretion/chromospheric lines}
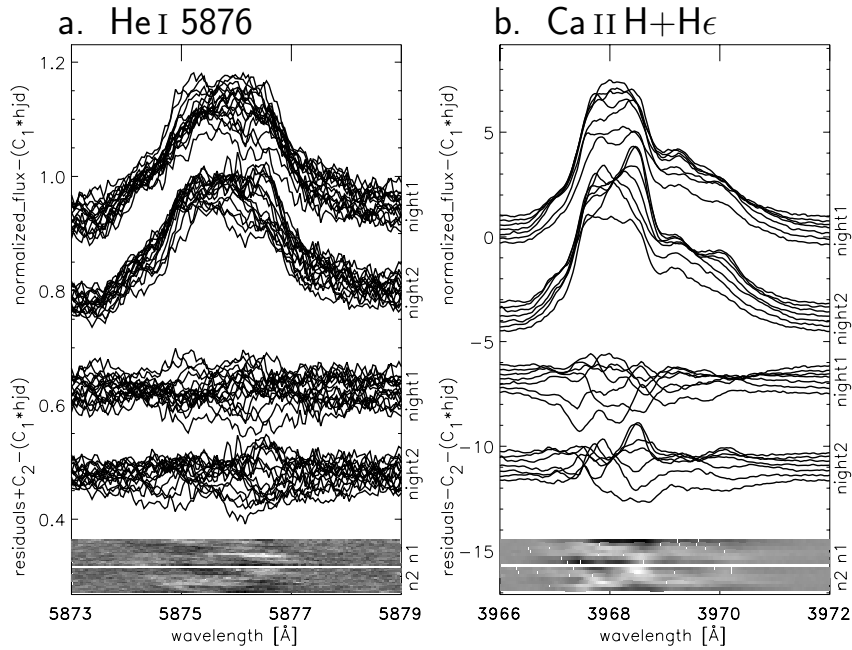

Fig. 6. Evolution of a) $\mathrm{He}$ I and b) $\mathrm{Ca}$ II $\mathrm{H}$ and $\mathrm{H} \epsilon$. Nomenclature as in Fig. 5.

these lines for a more direct mapping of the accretion shocks in a forthcoming paper because it involves three-dimensional structure that currently can not be handled by our inversion code TempMap.

The line width of the $\mathrm{He}-\mathrm{I}_{3}$ emission at $587.6 \mathrm{~nm}$ (Fig. 6a) is $240 \pm 20 \mathrm{~km} \mathrm{~s}^{-1}$, comparable with the Balmer emission lines except $\mathrm{H} \alpha$, which appears more broadened. The persistently strong He I emission suggests continuous high temperatures well above $10000 \mathrm{~K}$ at its location of origin. If we assume this region to be the accretion shock(s), its profile variations suggest permanent and spatially inhomogeneous impacts on the stellar surface. Its line-profile variability pattern is similar to the $\mathrm{H}$-lines or the $\mathrm{Ca}$ II $\mathrm{H}$ and $\mathrm{K}$ lines (Fig. 6). As with the Balmer lines, an additional chromospheric contribution is likely present but can not be separated from the data without explicit modelling.

Numerous weak and narrow emission lines appear with a double-peaked profile. The average width at continuum level of these lines is just $24 \mathrm{~km} \mathrm{~s}^{-1}$ (a fifth of the expected rotational broadening of MN Lupi). Its blue-to-red peak separation is even smaller, between $7-12 \mathrm{~km} \mathrm{~s}^{-1}$, close to the resolution limit of our observations of $\approx 5 \mathrm{~km} \mathrm{~s}^{-1}$. Their line intensities and equivalent widths vary consistently with air mass while their radial velocity remains constant. These lines are obviously of telluric origin as marked in Fig. 1 and are due to the airglow layer.

By far the strongest emission lines are the forbidden oxygen lines from [O I] at 557.7, 630.0, and $636.4 \mathrm{~nm}$ with line intensities relative to the continuum of up to 50,20 , and 8 , respectively (for comparison, $\mathrm{H} \alpha$ reaches at most 3; see also the T-Tauri star spectral atlas by Appenzeller et al. 1986). The width at continuum level is even smaller than for the weak and narrow telluric emission lines, on average just $13 \mathrm{~km} \mathrm{~s}^{-1}$ (a tenth of the expected photospheric rotational broadening). None of the three O I lines appear asymmetric or even double peaked as for most CTTSs (Edwards et al. 1987). The line intensities vary by almost $100 \%$ over the course of a night, without any variation of the radial velocity. While these changes are consistent for the 630.0-nm and 636.4-nm lines, the 557.7-nm line intensity seems to change inversely to the two other oxygen lines. The two strong and very narrow $\mathrm{Na} \mathrm{D}$ emission lines - that we interpret to be geocoronal in origin - behave the same way as [O I] $557.7 \mathrm{~nm}$, suggesting that almost all of the [O I] emission is also geocoronal in origin, in agreement with the findings of Hanuschik (2003). That interpretation agrees with the fact that we do not see any signs of the low-density sensitive [S II] or [N II] emissions at $671.6 \mathrm{~nm}, 673.1 \mathrm{~nm}$ and $654.8 \mathrm{~nm}$, $658.4 \mathrm{~nm}$, respectively, which suggests that MN Lupi does not have the typical loosely-collimated bipolar outflow. A partial background contamination due to diffuse [O I] emission from the Lupus cloud, or a H II region, can not be completely excluded.

\subsection{The absorption-line spectrum}

The analysis of photospheric CTTS spectra is hampered by the fact that the spectrum is diluted due to a pseudo continuum from a hot accretion shock. This decreases the depth of photospheric absorption lines by a factor that is called the "veiling" (e.g. Calvet \& Gullbring 1998).

Stempels \& Piskunov (2002) applied two different techniques to determine the veiling for the CTTS RU Lupi. We are following their approach and use our M 0 standard HD 209290 as a template star and solve for the veiling factor in a few wavelength ranges that are interesting for photospheric Doppler imaging. Furthermore, we use the Kurucz (1993) ATLAS-9 model atmospheres within our Doppler-imaging code to compute an optimal template line profile that best fits the line profile shape and the equivalent width of a number of relatively unblended spectral lines. For the Li I 670.8, the Ca I 643.9 and the Fe I 610.2 region, this yields slightly negative veiling factors $f_{\mathrm{v}}=\left(d-d^{\prime}\right) / d^{\prime}$ of a few per cent $\left(d^{\prime}\right.$ is the observed relative line depth, and $d$ the unveiled relative line depth). Therefore, we conclude that no veiling is present at these wavelengths and attribute the slightly negative veiling factors to a combination of relatively poor $\mathrm{S} / \mathrm{N}$ and the high degree of blending.

We have also measured the lithium equivalent width from the 670.8-nm line in all 33 spectra by integrating between two continuum points. Its average value is $394 \pm 15$ (rms) $\mathrm{m} \AA$. No lithium is detectable in our M 0 reference star HD 209290.

However, in MN Lupi we observe a number of triangularshaped absorption lines that appear neither in the atomic line lists, like VALD, nor in common molecular line lists. Figure 7 compares our time series of the neutral lithium line at $670.8 \mathrm{~nm}$ and the blend of a neutral iron line at $610.2 \mathrm{~nm}$ with the excited lithium line at $610.4 \mathrm{~nm}$. While the $670.8-\mathrm{nm}$ lithium resonance line shows a flat bottomed profile typical for spotted stars with polar or high-latitude spots, the iron line shows a triangular-shaped profile (with the excited lithium blend in the red wing). Further examples of such lines are $\lambda 638.16 \mathrm{~nm}$ with only a very weak V I line match, $\lambda 638.85 \mathrm{~nm}$ also with only a very weak Sr I line match, and $\lambda 644.98 \mathrm{~nm}$ which should be a Ca line but it is much stronger than predicted by TempMap or by R. Gray's synthesis program (for $T_{\text {eff }}=3750$ to 4250 ). 


\section{Photospheric absorption lines}
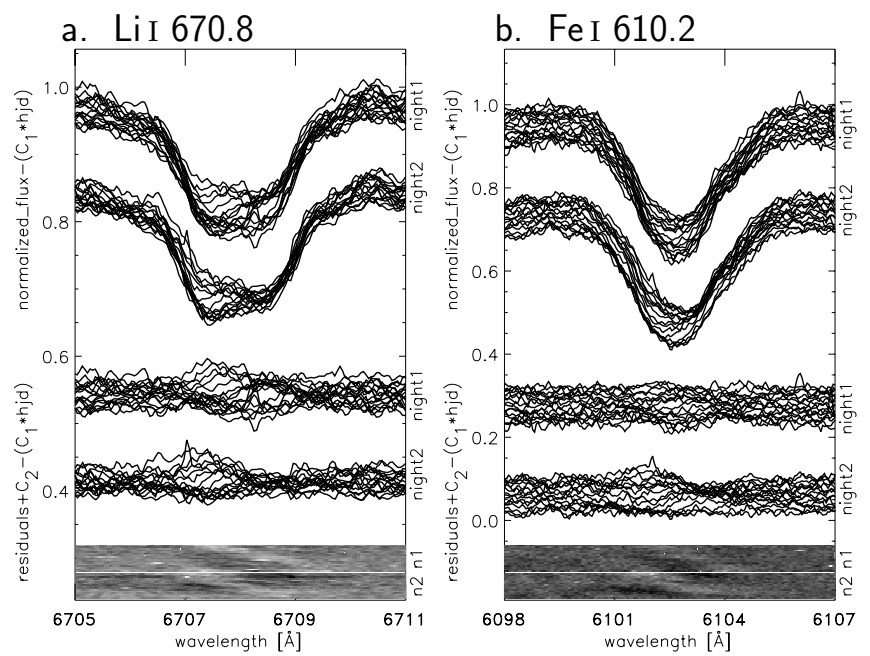

Fig. 7. Two examples for the evolution of photospheric absorption line profiles. a) Li I $670.8 \mathrm{~nm}$, b) Fe I $610.2 \mathrm{~nm}$. Nomenclature as in Fig. 5. Note that the Li line appears flat bottomed while the Fe line has a more triangular shape.

On the other hand, our standard imaging line Fe I $643.085 \mathrm{~nm}$ seems much weaker than it should be. Generally there are many lines of $\mathrm{Fe}$ and $\mathrm{Ca}$ that seem to have a more normal line shape but are severely blended. Most likely, the spectrum of MN Lupi consists of many weak molecular features that we can not readily identify at the given line broadening. We conclude that the $670.8 \mathrm{~nm}$ lithium line appears to be completely photospheric in origin and uncontaminated by molecular blends.

\subsection{Absolute dimensions}

Hughes et al. (1993) determined a distance to the Lupus starforming region of $140 \pm 20 \mathrm{pc}$ from the parallaxes of foreground stars while Hipparcos observed five pms stars in the Lupus region and its values suggest a distance of $190 \pm 27$ pc (Wichmann et al. 1998b). However, Wichmann et al. (1999) reevaluated the distance with the assumption of known rotational periods, line broadenings, and inclinations for the Li-rich stars in the Lupus region on a statistical basis. They found the most likely distance to be 135-165 pc, only in moderate agreement with the Hipparcos parallax. We adopt their average value of $150 \pm 15 \mathrm{pc}$ in this paper.

The "least-spotted" magnitude of MN Lupi, i.e. the magnitude at the peak of the light curve, is $V=14 \mathrm{~m} 13 \pm 0.03$ (see Sect. 3.1) which converts, using a distance of $150 \pm 15 \mathrm{pc}$ and $A_{\mathrm{V}} \approx 0.74$ (see Sect. 3.1), to $M_{V}=8$.99. With a bolometric correction of -1.38 and a bolometric magnitude for the Sun of +4.74 (Cox 2000) one gets a bolometric magnitude of $6.87 \pm 0.21$ for MN Lupi which is equivalent to a luminosity of $0.14 \pm 0.02 L_{\odot}$. These results then imply a radius of $0.87 \pm 0.06 R_{\odot}$ for $\mathrm{MN}$ Lupi. Note that Table 2 summarizes the parameters that are later also used in the Doppler-imaging analysis.

Figure 8 shows the position of MN Lupi in the H-R diagram. We adopted the effective temperature of $3800 \pm 150 \mathrm{~K}$
Table 2. Astrophysical data for MN Lupi.

\begin{tabular}{ll}
\hline \hline Parameter & \\
\hline Distance & $150 \mathrm{pc}$ (adopted) \\
Spectral type & $\mathrm{M} 0$ \\
$M_{\mathrm{V}}$ & +8.99 \\
Luminosity & $0.15 \pm 0.03 L_{\odot}\left(\right.$ from $\left.M_{V}\right)$ \\
& $0.14 \pm 0.02 L_{\odot}\left(\right.$ from $R_{v \sin i}$ and $\left.T_{\text {eff }}\right)$ \\
$\log g$ & 4.5 (adopted) \\
$T_{\text {eff }}$ & $3800 \pm 150 \mathrm{~K}$ \\
Radius & $0.87 \pm 0.06 R_{\odot}\left(\right.$ from $L$ and $\left.T_{\text {eff }}\right)$ \\
& $0.90 \pm 0.02 R_{\odot}($ from $v \sin i$ and $i)$ \\
$v$ sin $i$ & $74.6 \pm 1.0 \mathrm{~km} \mathrm{~s}{ }^{-1}$ \\
Rotation period & $0.439 \pm 0.005$ days $(10.5 \mathrm{~h})$ \\
Minimum radius & $0.648 \pm 0.015 R_{\odot}($ from $P$ and $v \sin i)$ \\
Inclination & $46 \pm 1^{\circ}($ from Doppler imaging $)$ \\
Mass & $0.68_{-0.10}^{+0.06} M_{\odot}($ Baraffe et al. tracks $)$ \\
& $0.54 \pm 0.12 M_{\odot}($ Siess et al. tracks $)$ \\
Age & $20 \pm 10 \mathrm{Myr}$ \\
Lithium abundance & $3.1 \pm 0.1(\log n(\mathrm{H})=12)$ \\
Macroturbulence, $\zeta$ & $1.0 \mathrm{~km} \mathrm{~s}^{-1}$ \\
Microturbulence, $\xi$ & $0.5 \mathrm{~km} \mathrm{~s}^{-1}$ \\
\hline &
\end{tabular}

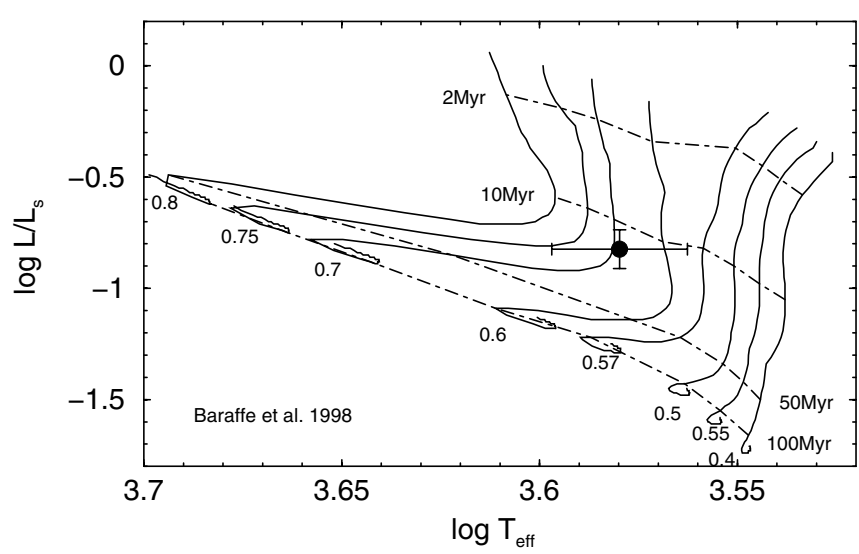

Fig. 8. MN Lupi in the H-R diagram. The dot with error bars is the position of MN Lupi. The full lines are the evolutionary tracks from Baraffe et al. (1998) for masses between 0.4-0.8 solar masses. The dash-dotted lines are the isochrones for 2, 10, 50, and $100 \mathrm{Myr}$. The comparison suggests a mass for MN Lupi of $0.68_{-0.10}^{+0.06} M_{\odot}$ and an age of $\approx 20$ Myr.

from the Doppler maps with rather conservative error estimates. The pre-main-sequence tracks are from Baraffe et al. (1998) for solar abundances. This comparison suggests a mass for MN Lupi of $0.68_{-0.10}^{+0.06} M_{\odot}$ and an age of 10-30 Myr $(\approx \log t$ of 7.30). Hartmann et al. (1998) list only one other T Tauri star with a disk at that age (HN Tau, $\log t \approx 7.5$ ). We note though that the various pre-main-sequence tracks found in the literature can give quite different estimates. Granzer (2005) compared various theoretical tracks based on different convection treatment, boundary conditions, and equations of state and found differences for a $0.6 M_{\odot}$ model of up to $10 \%$ in $\Delta T_{\text {eff }} / T$ 
and $25 \%$ in $\Delta L / L$. For example, when using the Siess et al. (2000) tracks, MN Lupi has a mass of $0.54 \pm 0.12 M_{\odot}$ but still about the same age (note that a normal main-sequence M 0 star is expected to have a mass of $0.51 M_{\odot}$ ).

A new value for $v \sin i$ of $74.6 \pm 1.0 \mathrm{~km} \mathrm{~s}^{-1}$ is derived from the Doppler imaging analysis (Sect. 4.5) and, together with the rotation period of $0.439 \pm 0.005$ days, suggests a minimum radius of $0.648 \pm 0.015 R_{\odot}$. The error mostly reflects the error of the $v \sin i$-measurement. Our Doppler images achieve good agreement within a range of inclinations of $\approx 40-50^{\circ}$ and formally give $i=46 \pm 1^{\circ}$ as the most consistent value based on $\chi^{2}$ minimization. This suggests a radius of $0.90 \pm 0.02 R_{\odot}$ as the most likely radius of MN Lupi, in agreement with the radius from the adopted distance. The luminosity of MN Lupi is then $0.15 \pm 0.03 L_{\odot}$. Note that a main-sequence M 0 star has a nominal radius of $0.60 R_{\odot}$, already smaller than the above minimum radius, while a $\mathrm{K} 7 \mathrm{~V}$-star has $0.65 R_{\odot}$, according to the tables of Gray (1992) and Cox (2000).

\section{Doppler imaging of MN Lupi}

\subsection{The TempMap code}

TempMap follows the standard mathematical process of Doppler imaging which is essentially a matter of creating an error function that represents the degree to which the predicted spectrum from a current trial image of a star (the forward calculation) differs from the observed spectrum and then altering the parameters of the image to iteratively reduce the error function to a minimum consistent with the error of the observations. Typically the error function is represented as

$E=\sum_{\phi} \sum_{\lambda} \frac{1}{\sigma_{\lambda, \phi}^{2}}\left[R_{\mathrm{calc}}(\lambda, \phi)-R_{\mathrm{obs}}(\lambda, \phi)\right]^{2}+\alpha f$.

Here $R_{\text {calc }}$ represents the predicted line spectrum from the forward calculation and $R_{\mathrm{obs}}$ represents the observed spectrum. The $\sigma_{\lambda, \phi}$ represents the error of the individual observations. The squared difference between the forward calculation and the observation is summed over all wavelengths and a full set of observations spaced in time through the full cycle of phases, $\phi$, of the star's rotation. The last term is used for regularization of the minimization process so that the line profiles calculated from the image do not overfit the observed spectra. For a fuller discussion of Doppler Imaging and the process of regularization see Rice (2002), and for extensive tests of the code see Rice \& Strassmeier (2000).

In TempMap, the local line profiles are calculated from Kurucz (1993) model atmospheres that are appropriate to the local conditions for each surface element of the image, conditions such as the effective temperature. A file of temperatures covering $3500 \mathrm{~K}$ to over $6500 \mathrm{~K}$ is provided for the purpose of recovering the local effective temperatures. Calculating the line profiles for every element of the surface for each iteration of the conjugate gradient minimization routine would demand an inordinate amount of computing time, so the profile calculations are tabled for an array of limb angles and temperatures as outlined in Piskunov \& Rice (1993).
With the expansion of computing power, TempMap now fits not just the time dependent variations of a single line but instead recovers images from multiple lines in the observed spectrum. In the present paper, the images are derived from single observed lines but the forward calculation of the local line profiles includes the blended effect of all reasonably strong lines that are found in the VALD listing (Piskunov et al. 1995; Kupka et al. 1999) at wavelengths near the principle line.

\subsection{Limiting spatial resolution due to phase smearing}

With a resolving power of UVES of $\lambda / \Delta \lambda=60000$ $\left(\Delta \lambda\right.$ of $0.0108 \mathrm{~nm}$ at $642.5 \mathrm{~nm}$, or $\approx 5 \mathrm{~km} \mathrm{~s}^{-1}$ ) and a full width of the photospheric absorption lines at continuum level of $2(\lambda / c) v \sin i \approx 0.32 \mathrm{~nm}$, we have $\approx 30$ resolution elements across the stellar disk. This is equivalent to a spatial surface resolution along the equator at the stellar meridian of approximately $7^{\circ}$. However, the total time-on-target of 35 min causes phase smearing due to stellar rotation of up to a maximum of 0.055 or $\approx 20^{\circ}$ along the stellar equator and in the direction of the rotational motion, which is three times the spectroscopic resolution limit. Our inversion code produces a solution that best agrees with the data, and thus implicitly always reconstructs a time-averaged image that is still consistent with the data. This must be taken into account when interpreting our images.

\subsection{Adopted rotation period}

Because the rotational period of the star is a pre-determined and fixed value for the line-profile inversion, if the period is wrong in the first place then the resulting images will also be wrong. We first computed trial images from Li I $670.8 \mathrm{~nm}$ for night $n 1$ for all periods that were evident in the photometry. In spite of the fact that the CLEAN periodogram suggested only the 0.439 -day period to be correct, the Lomb-Scargle periodogram (Sect. 3.2) had two further frequency peaks at periods of 0.31 days $\left(f_{0}+1\right)$ and 0.78 days $\left(f_{0}-1\right)$. None of the maps with 0.31 and 0.78 days arranged the line profiles such that their changes made sense if they were due to rotation, i.e., sometimes profile bumps were followed by dips and again by bumps. The maps appeared correspondingly messy having an overall $\chi^{2}$ never better than a factor of two of the ones with 0.439 days. We conclude that the 0.439 -day period is indeed the correct one.

\subsection{Oblateness of the star?}

Because the star rotates at $27 \%$ of its break-up velocity it will be rotationally distorted. However, the ratio of the polar radius to the equatorial radius, $R_{\text {pole }} / R_{\text {equ }}$, is just 0.965 . This value is obtained from the assumption of equal Roche potentials at the equator and the pole, following the prescription in Slettebak (1949), together with a mass of $0.68 M_{\odot}$ and an equatorial radius of $0.89 R_{\odot}$ (the latter is a weighted average of the two methods in Sect. 3.6). The gravity ratio equator-to-pole is 0.931 and converts to a equator-to-pole temperature gradient of $22 \mathrm{~K}$ 

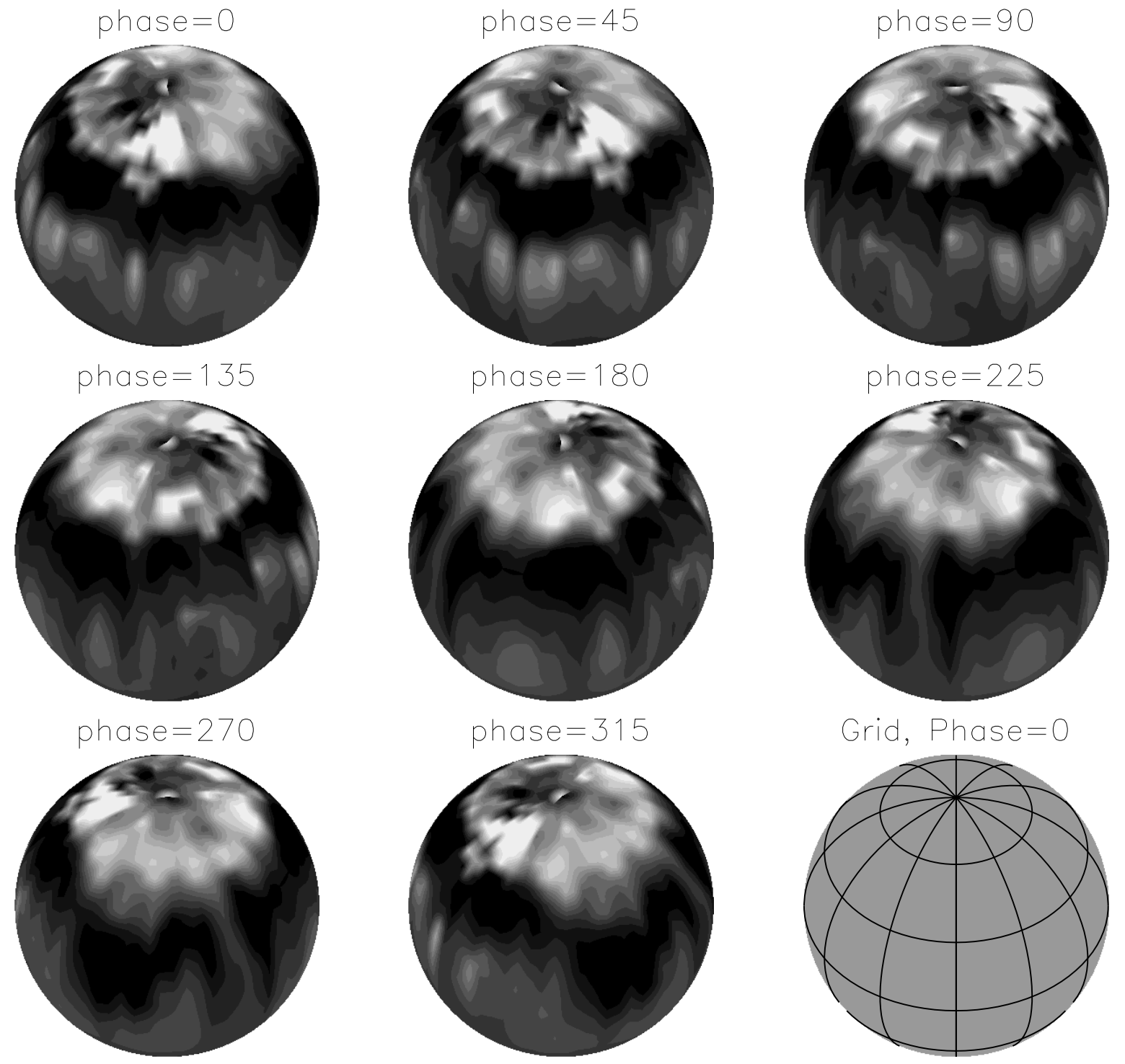

$3370 \mathrm{~K}$

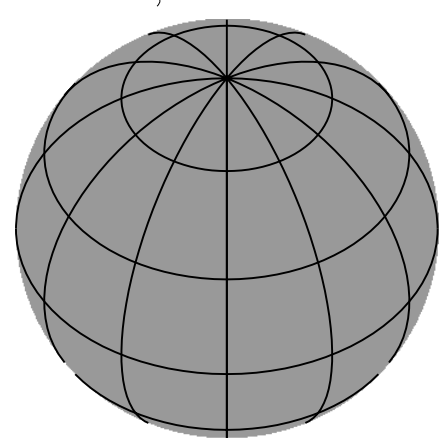

$5800 \mathrm{~K}$

Fig. 9. Doppler image of MN Lupi for May 27, 2000 (image $n 1$ ). Maps are plotted in a spherical projection at eight equidistant rotational phases. The temperature scale is indicated and is the same for all projections. The photospheric temperature is $3800 \mathrm{~K}$. A ring of higher temperature around the visible rotational pole suggests remnant heating of the photosphere from accretion shocks. Several hot spots are identified and document the inhomogeneity of the accretion process.

from von Zeipel's $T \propto g^{0.08}$ law (Zeipel 1946; with a gravitydarkening exponent of 0.08 for stars with a convective envelope and with $T_{\text {eff }}=T_{\text {equ }}=3800 \mathrm{~K}$ ). This is several times below the resolution capability of our data. Nevertheless, we carried out numerical simulations with a modified version of TempMap which accounts for a rotationally distorted stellar surface (see Kővári et al. 2006). We pre-fix the oblateness, defined as $\left(1-\left(R_{\text {pole }} / R_{\text {equ }}\right)^{2}\right)^{0.5}$, invert the data and then go through a number of trial oblatenesses and search for the lowest $\chi^{2}$ and for systematic surface morphology differences. We applied this procedure to both data sets of MN Lup by assuming two different values of the radius ratio, 0.965 and an arbitrary 0.92. From these tests it became obvious that even the relatively large ratio of 0.92 had no significant impact on our line-profile reconstruction. The achievable $\chi^{2}$, at the $\mathrm{S} / \mathrm{N}$ of the data given, is also practically indistinguishable from the spherical case. Therefore, we conclude that gravity darkening and rotational deformation of MN Lupi can be neglected for our Doppler imaging.

\subsection{Results}

Figures 9 and 10 show the final images from Li I $670.8 \mathrm{~nm}$ for the two consecutive nights, respectively. The observed spectral profiles and their respective fits are shown in Fig. 11. The lithium line was inverted as is, i.e., without applying any veiling and with a fixed lithium abundance of $\log n(\mathrm{Li})=3.1 \pm 0.1$ (on the usual $\log n(\mathrm{H})=12$ scale). This lithium abundance was 

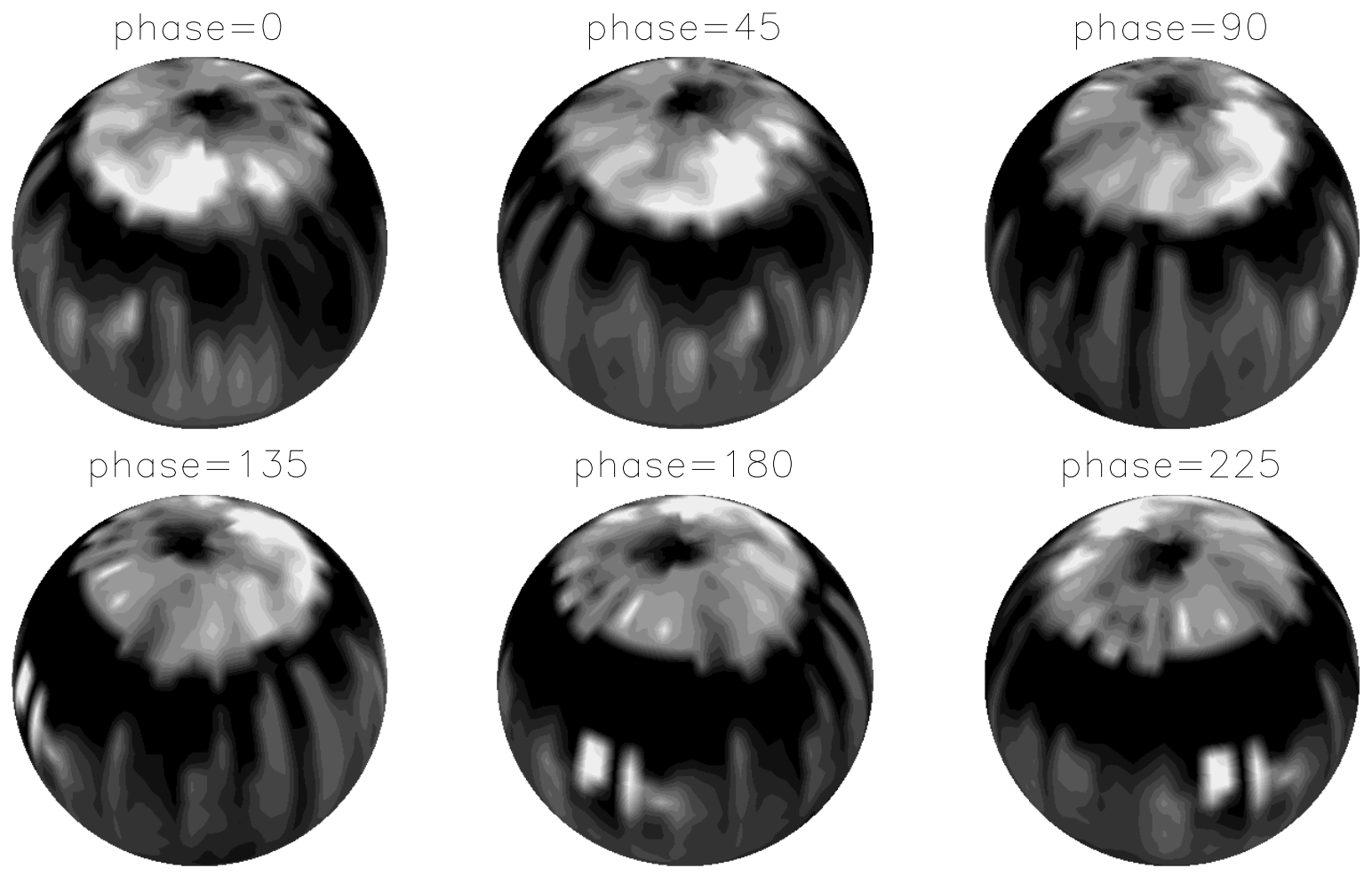

phase $=270$
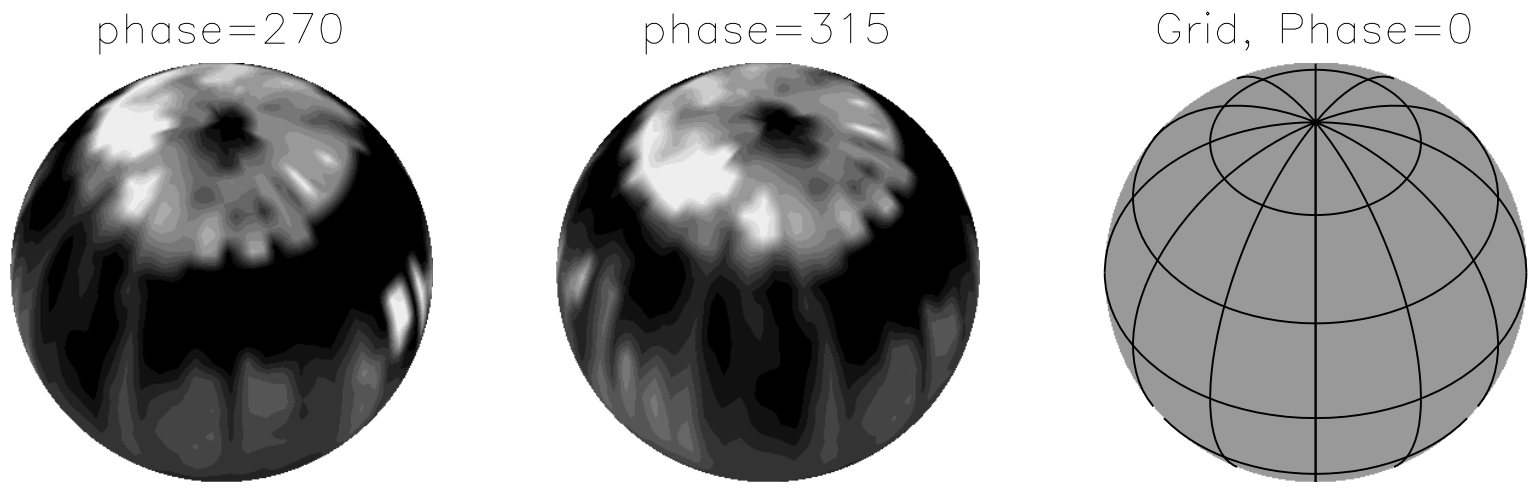

$3370 \mathrm{~K}$

$5800 \mathrm{~K}$

Fig. 10. Doppler image of MN Lupi for May 28, 2000 (image $n 2$ ), separated from image $n 1$ by 14 h or 1.3 stellar rotations. Although the overall surface morphology remained as in image $n 1$, some structures seem to have changed.

obtained from the best compromise in the parameter space abundance, $v \sin i$, micro- and macroturbulence, transition probabilities (a.o.), and is typical for pre-main-sequence stars.

Both maps show a structured, warm $(5000 \mathrm{~K})$, ring-like band around the visible rotational pole. The band's locus is possibly off centered by $\approx 10^{\circ}$ towards longitude $90^{\circ}$ in image $n 1$, but towards $270^{\circ}$ in image $n 2$. A central opening in image $n 2$ appears $400 \mathrm{~K}$ cooler than the effective temperature of $3800 \mathrm{~K}$, but could be partially artificial due to the limited velocity information near the pole. During both nights two hot regions are seen within the warm band; a large monolithic one centered at longitude $0^{\circ}$ and latitude $65^{\circ}$ in both images and a close double spot centered at $\approx 160^{\circ}$ in image $n 1$ at a latitude of $+65^{\circ}$, and $200^{\circ}$ and on the stellar equator in image $n 2$ (all of them with an average temperature of $5800 \mathrm{~K}$ ). The former region appears very similar in both images, even in shape. The latter seems to have either shifted in longitude and significantly decreased in size (the latter by a factor of four; compare the images at longitude $\approx 180^{\circ}$ in Figs. 9 and 10 , respectively) and/or was wrongly reconstructed and partly mirrored to the equator. Note that image $n 2$ recovered a weak mirror image of the double equatorial hot spot at the latitude within the warm band where it was seen the night before in image $n 1$. While the two hot regions are associated with the sharp profile valleys seen in the data at longitudes $189^{\circ}$ and $185^{\circ}$ for $n 1$ and $n 2$, respectively (cf. Figs. 11a and 11b), we can not visually identify the double structure of the latter in the line profiles. The profile at longitude $226^{\circ}$ in Fig. 11 b may indicate two close "valleys" between two "peaks", indicative of a double structure. However, the current data do not allow a clean decision regarding the true location of this particular feature during the second night and we do not further discuss it. 
a. Data and fits for image $n 1$
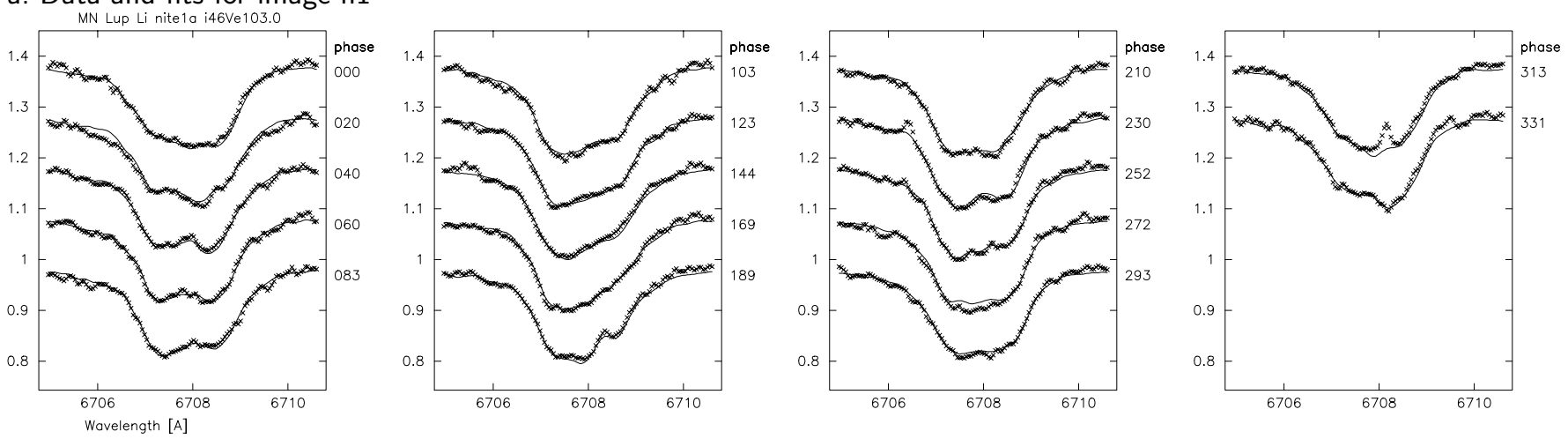

b. Data and fits for image n2
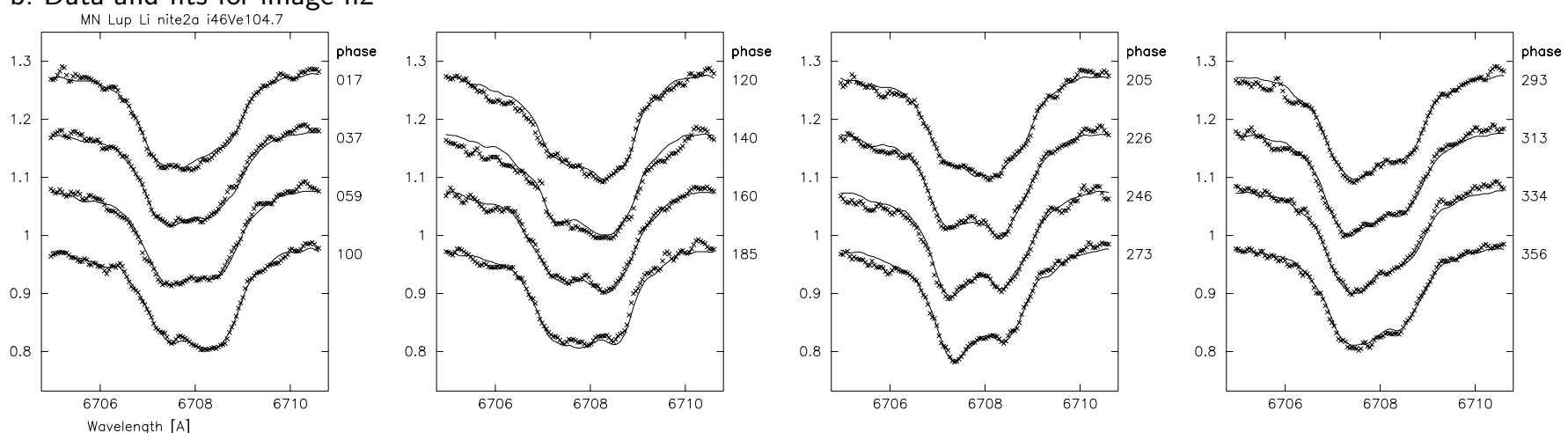

Fig. 11. Observations (crosses) and fits (lines) from the inversion of the Li I 670.8-nm profiles for a) night $n 1$ and b) night $n 2$. Note that the pixels with the cosmic-ray hit in profile $n 1 / 313$ were given zero weight in the inversion.

The lower rim of the warm band is immediately followed by a structured cool (3400-3500 K) band stretching to below the stellar equator and all around the star. It is of varying width and has its smallest part of just $\approx 10^{\circ}$ crossing the central meridian roughly at phase $90^{\circ}$ and its thickest part of $\approx 40^{\circ}$ roughly at phase $200^{\circ}$. No truly reliable information can be retrieved below the stellar equator due to the phase smearing of our data. Also note that the latitudinal blurring at and below the equator is likely not real and due to the still imperfect phase coverage. Although such imperfect phase coverage is treated implicitly some residual uncertainty whether a flux element belongs to a certain latitude or not remains.

A cross correlation of the two images (see Kővári et al. 2004 for a description of the method) reveals a high degree of correlation for the features within the high-latitude warm band between $50-70^{\circ}$ latitude but almost none for the lowlatitude/equatorial cool band. This may be evidence that the low-latitude spots are mostly artificial and that the accretion disk actually blocks the photospheric light from parts of the lower hemisphere.

Only two more CTTSs have been Doppler imaged to date. DF Tau (Unruh et al. 1998) and SU Aur (Unruh et al. 2004; Petrov et al. 1996). SU Aur is a much more massive, hotter and larger system $\left(2-2.5 M_{\odot}, 5700 \mathrm{~K}, 10 L_{\odot}\right)$ at an age of $\approx 3 \mathrm{Myr}$. Therefore, its Doppler maps can not be directly compared with our MN Lupi maps. We also note that the Doppler-imaging code applied by Unruh et al. could reconstruct either hot or cool spots and that three very large hot $(6000 \mathrm{~K})$ spots were reconstructed when only hot spots were allowed but a large cool polar cap was reconstructed when only cool spots were allowed. DF Tau, on the other hand, is a M2 CTTS binary but with a significantly longer rotation period of 8.5 days and also much more massive than MN Lupi. Again, using the same Doppler imaging code as for SU Aur the authors reconstructed at least two very large and hot $(5500 \mathrm{~K})$ spots, one on the equator and one at mid latitudes. Generally, DF Tau is a highly variable star and its luminosity determinations range from $1.7 L_{\odot}$ to $6.3 L_{\odot}$ from various authors, implying a stellar radius of 3 to $6 R_{\odot}$, thus also quite different than MN Lupi.

\section{A magnetic star-disk model for MN Lupi}

The spin down of an accreting star by its surrounding accretion disk was first proposed by Ghosh \& Lamb (1979a,b) for the spin-down of neutron stars. The model was later adopted to explain the moderate rotation rates of T Tauri stars (Camenzind 1990; Königl 1991; Shu et al. 1994; Armitage \& Clarke 1996). In a recent paper, Matt \& Pudritz (2005) argue that the diskbraking mechanism becomes less efficient if the finite extent of the region of the disk that is connected to the star is taken into account. As a result the spin-down time scale increases by about an order of magnitude, depending on the field geometry.

In their model the equilibrium rotation frequency is

$\Omega_{\mathrm{eq}}=C\left(\beta, \gamma_{\mathrm{c}}\right) \dot{M}_{\mathrm{a}}^{3 / 7}\left(G M_{*}\right)^{5 / 7} \mu^{-6 / 7}$, 
where $\dot{M}_{\mathrm{a}}$ is the mass accretion rate, $G$ is Newton's constant of gravity, $M_{*}$ the stellar mass, and $\mu=R_{*}^{3} B_{*}$ the stellar dipole moment. $C(\beta)$ is a function of the diffusion parameter,

$\beta=\frac{\alpha h}{r \mathrm{Pm}}$,

where $\alpha$ is the Shakura-Sunyaev viscosity parameter, $h$ the height of the disk, Pm the magnetic Prandtl number, and $r$ the radius. The parameter $\gamma=B_{\phi} / B_{r}$ describes the field geometry and $\gamma_{\mathrm{c}}$ is the maximum $\gamma$ for a stable field configuration. With a mass accretion rate of $10^{-9} M_{\odot} \mathrm{yr}^{-1}$ (adopted), a stellar mass of $0.68 M_{\odot}$ from Sect. 3.6, a stellar radius of $0.90 R_{\odot}$ (also from Sect. 3.6), and a magnetic field strength of $1 \mathrm{kG}$ (adopted) at the poles we find

$\Omega_{\mathrm{eq}}=2.8 \times 10^{-5} s^{-1} C$,

or

$P_{\text {eq }}=\frac{2.6 \text { days }}{C\left(\beta, \gamma_{\mathrm{c}}\right)}$

for the equilibrium rotation period. Assuming $\gamma_{\mathrm{c}}=1$, the value of $C$ varies between 3 and 12 in the interval $0.01 \leq \beta \leq 100$, giving an estimate for the rotation period of

$$
0.2 \lesssim P_{\text {eq }} \lesssim 1 \text { days. }
$$

The observed rotation period of 0.44 days lies well within this interval. Applying their model to BP Tau, Matt \& Pudritz (2005) argue, however, that the dipolar component of the stellar magnetic field is probably much weaker, of the order $100 \mathrm{G}$ rather than $1 \mathrm{kG}$. In that case the truncation radius of the disk would lie too close to the star for disk locking to be even possible. A smaller mass accretion rate of $10^{-11} M_{\odot} \mathrm{yr}^{-1}$, on the other hand, would be compatible with a polar field strength of $100 \mathrm{G}$.

The conditions for the formation of a funnel flow turn out to be harder to meet than those for disk locking. Miller \& Stone (1997) studied the interaction between a star-centered dipole field and a circumstellar accretion disk for different field geometries. They found that a funnel flow only occurs if the magnetic pressure of the stellar dipole field is large enough to balance the ram pressure of the gas in the disk. This finding was confirmed by Romanova et al. (2003). Figure 12 shows a snapshot of MN Lupi computed from a modified version of the model by Küker et al. (2003). The main difference lies in the choice of the inner boundary, which is now the stellar surface. The magnetic diffusion term was dropped, leaving only numerical diffusion and viscosity. At the beginning of the simulation the disk is truncated at the corotation radius. Viscous and magnetic stress then brake the rotation of the gas, which thus moves inwards and the inner radius of the disk moves towards the star. The magnetic pressure rapidly increases with decreasing distance from the star and a point is finally reached where the gas can no longer move radially inwards. It then piles up at that radius and eventually a gas flow evolves along the magnetic field, which hits the stellar surface at high latitudes $\left(\approx 50^{\circ}\right)$. The position of the inner disk radius is thus determined by the balance between magnetic pressure and mass accretion. For an accretion rate of $10^{-11} M_{\odot} \mathrm{yr}^{-1}$ we find that a field strength of $3 \mathrm{kG}$

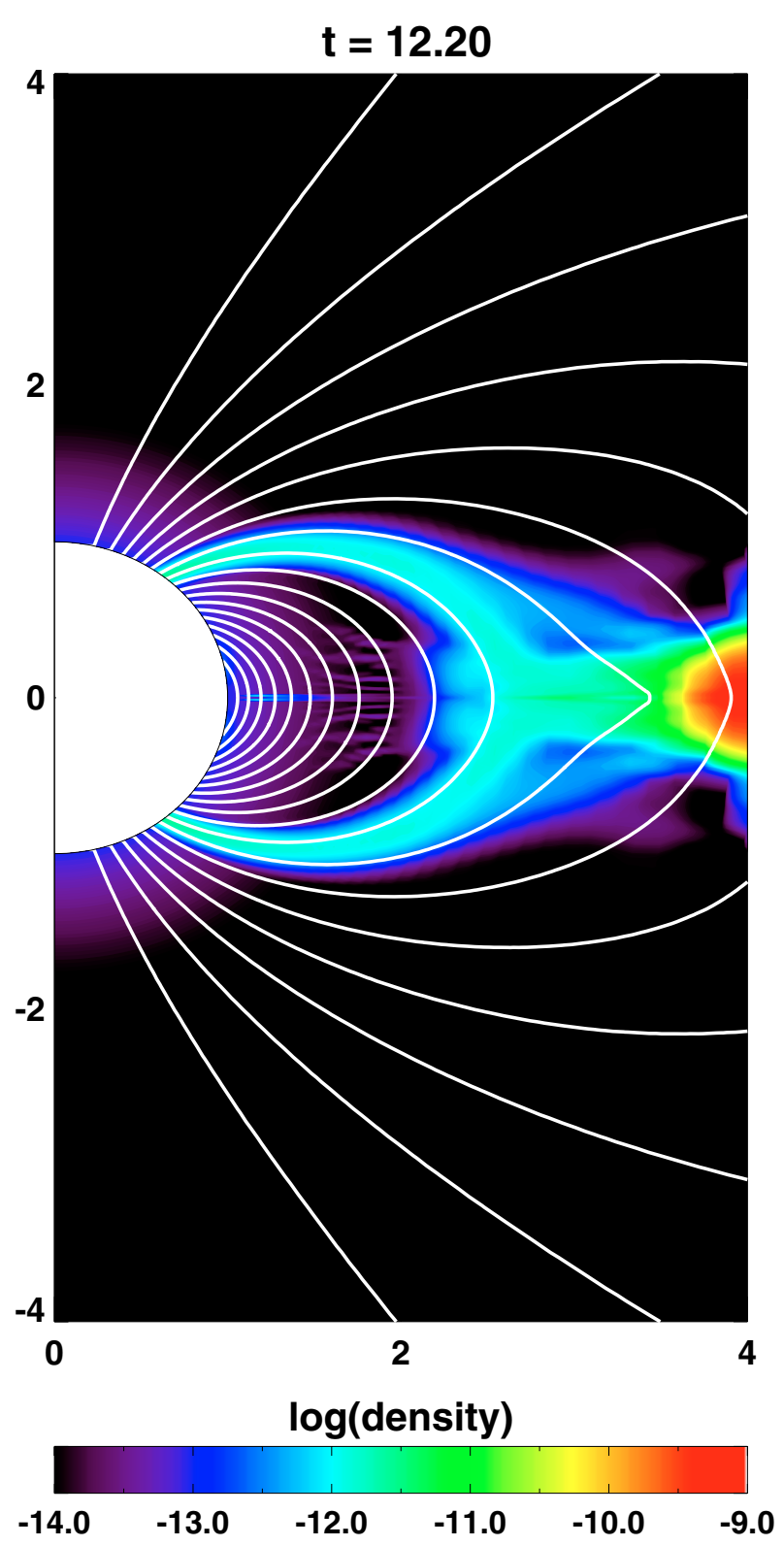

Fig. 12. Poloidal field lines and density contours for the inner part of the MN Lupi system (units in stellar radii). The outer boundary is located at $r=20$. The time unit is the stellar breakup rotation period. The initial disk was computed from a standard model with a viscosity parameter $\alpha=0.01$, an accretion rate of $10^{-11} M_{\odot} \mathrm{yr}^{-1}$ and a Kramers-type opacity law. The (dipole) field strength on the stellar surface is $3 \mathrm{kG}$ at the poles.

is necessary to enforce funnel flow. For smaller strengths of the stellar dipole field the disk extends down to the stellar surface. In this case the accretion torque is balanced by the magnetic torque while the viscous torque becomes marginal. Larger accretion rates require stronger magnetic fields. For an accretion rate of $10^{-9} M_{\odot} \mathrm{yr}^{-1}$ we found a value of about $15 \mathrm{kG}$ (polar field strength on the stellar surface) to be necessary. These large values of the field strength lead to a distortion and partial disruption of the inner part of the disk up to about four corotation radii and the gas flow is strongly non stationary. 


\section{Discussion and conclusions}

The rotation rate of MN Lupi of $10.5 \mathrm{~h}$ is just 3.7 times below its break-up rotation rate of $2.8 \mathrm{~h}$. It is therefore among the fastest rotators of non-degenerate stars. The simultaneous presence of high-excitation Balmer lines and periodically changing broadened photospheric absorption lines hint towards a complex evolution of the magnetic star-disk coupling and its associated angular-momentum exchange. MN Lupi seems to be an example of a CTTS that has been spun up recently, or is still being spun up, while accretion onto the stellar surface is ongoing at the same time. The commonly referred to "dipolar magnetospheric accretion model" (cf. Ghosh \& Lamb 1979a,b) predicts a toroidal field component from the rotational shear between the star and the disk that reverses its sign at the corotation radius, as does the torque exerted on the disk. Inside the corotation radius, angular momentum is transferred along these field lines from the disk to the star, and thus spins up the star. Outside the corotation radius the disk acts to brake the stellar rotation. The 2-D MHD models of Küker et al. (2003) for a solar-mass CTTS with a $1 \mathrm{kG}$ surface field suggest that the accretion torque exceeds the magnetic torque by two orders of magnitude. Therefore, the total torque at the inner boundary of the disk would be always negative and consequently spin up the stellar surface long before the disk is depleted. At a field of as strong as $10 \mathrm{kG}$ the disk will be disrupted out to radii beyond the corotation radius.

Indeed, classical T Tauri stars are observed to have strong magnetic fields on their surface as well as within their accretion shocks slightly above the surface, as inferred from circularly polarized He I line emission. For a list of existing magnetic field measures of CTTSs see Johns-Krull et al. (2003). Their compilation of 13 CTTSs suggests surface fields between $1.0-2.7 \mathrm{kG}$, and field strengths in the shock regions of between $1.0-2.9 \mathrm{kG}$, with the bulk of stars having field strengths of $\approx 2.5 \mathrm{kG}$. This led Johns-Krull \& Gafford (2002) to assume that all CTTSs have similar field strengths, perhaps due to a dynamo operating at its limit.

We interpret the hot spots in our Doppler images as due to the accretion shocks from material that is funnelled along magnetic field lines. The model of Küker et al. then predicts a (dipole) field strength for MN Lupi at the poles at the stellar surface of $\approx 3 \mathrm{kG}$.

A direct measurement of the magnetic field of MN Lupi is clearly needed and would further constrain our magnetic stardisk model. Stars with a visual magnitude of as faint as 14.3 are currently not within reach for Zeeman Doppler imaging though, but will be prime targets for PEPSI, the upcoming highresolution spectropolarimeter for the $11.8 \mathrm{~m}$ Large Binocular Telescope in southern Arizona (Strassmeier et al. 2004) - just to make an optimistic statement for the future.

Acknowledgements. K.G.S. is very grateful to the German Science Foundation (DFG) for support under grant STR645. We thank Scott Wolk and Bo Reipurth for discussions about CTTS accretion geometry and Thomas Granzer for computing the stellar models for us and an anonymous referee for helping to improve the paper.

\section{References}

Appenzeller, I., Jetter, R., \& Jankovics, I. 1986, A\&AS, 64, 65

Armitage, P. J., \& Clarke, C. J. 1996, MNRAS, 280, 458

Baraffe, I., Chabrier, G., Allard, F., \& Hauschildt, P. H. 1998, A\&A, 337,403

Bertout, C. 1989, ARA\&A, 27, 351

Bessell, M. S. 1979, PASP, 91, 589

Bessell, M. S. 1990, A\&AS, 83, 357

Bessell, M. S., Castelli, F., \& Plez, B. 1998, A\&A, 333, 231

Calvet, N., \& Gullbring, E. 1998, ApJ, 509, 802

Camenzind, M. 1990, RvMA, 3, 234

Cox, A. N. (ed.) 2000, Allen's Astrophysical Quantities, 4th edition (New York Berlin Heidelberg: Springer Verlag)

Dame, T. M., Ungerechts, H., Cohen, R. S., et al. 1987, ApJ, 322, 706

D'Antona, F., \& Mazzitelli, I. 1997, in Cool stars in clusters and associations, ed. R. Pallavicini, \& G. Micela, Mem. S.A. It. 68, 807

Dekker, H., D’Odorico, S., Kaufer, A., Delabre, B., \& Kotzlowski, H. 2000, Proc. SPIE, 4008, 534

Donati, J. F., Mengel, M., \& Carter, B. D. 2000, MNRAS, 316, 699

Dubath, P., Reipurth, B., \& Mayor, M. 1996, A\&A, 308, 107

Edwards, S., Cabrit, S., Strom, S. E., et al. 1987, ApJ, 321, 473

Flower, P. J. 1996, ApJ, 469, 355

Ghosh, P., \& Lamb, F. K. 1979a, ApJ, 232, 259

Ghosh, P., \& Lamb, F. K. 1979b, ApJ, 234, 296

Granzer, T. 2005, in 13th Cool Stars, Stellar Systems, and the Sun, Hamburg, ed. Favata et al., ESA-SP, 560, 591

Gray, D. F. 1992, The observation and analysis of stellar photospheres, CUP, 431

Gullbring, E., Hartmann, L., Briceno, C., \& Calvet, N. 1998, ApJ, 492, 323

Hanuschik, R. W. 2003, A\&A, 407, 1157

Hartigan, P., Edwards, S., \& Ghandour, L. 1995, ApJ, 452, 736

Hartmann, L., Boss, A., Calvet, N., \& Whitney, B. 1994, ApJ, 430, L49

Hartmann, L., Calvet, N., Gullbring, E., \& D’Alessio, P. 1998, ApJ, 495, 385

Hughes, J., Hartigan, P., \& Clampitt, L. 1993, AJ, 104, 680

Johns-Krull, C. M., \& Gafford, A. D. 2002, ApJ, 573, 685

Johns-Krull, C. M., Valenti, J. A., \& Gafford, A. D. 2003, Rev. Mex. Astron. Astrofis., 18, 38

Königl, A. 1991, ApJ, 370, L39

Kővári, Zs., Strassmeier, K. G., Granzer, T., et al. 2004, A\&A, 417, 1047

Kővári, Zs., Bartus, J., Strassmeier, K. G., et al. 2006, A\&A, in preparation

Krautter, J., Wichmann, R., Schmitt, J. H. M. M., et al. 1997, A\&AS, 123,329

Küker, M., Henning, T., \& Rüdiger, G. 2003, ApJ, 589, 397

Kupka, F., Piskunov, N. E., Ryabchikova, T. A., Stempels, H. C., \& Weiss, W. W. 1999, A\&AS, 138, 119

Kurucz, R. L. 1993, ATLAS-9, CD-ROM \#13

Matt, S., \& Pudritz, R. E. 2005, MNRAS, 356, 167

Miller, K. A., \& Stone, J. M. 1997, ApJ, 489, 890

Nidever, D. L., Marcy, G. W., Butler, R. P., Fischer, D. A., \& Vogt, S. S. 2002, ApJS, 141, 503

Pavlenko, Ya. V., \& Magazzú, A. 1996, A\&A, 311, 961

Petrov, P. P., Gullbring, E., Ilyin, I., et al. 1996, A\&A, 314, 821

Piskunov, N. E., \& Rice, J. B. 1993, PASP, 105, 1415

Piskunov, N. E., Kupka, F., Ryabchikova, T., Weiss, W., \& Jeffery, C. 1995, A\&AS, 112, 525

Rice, J. B. 2002, AN, 323, 220

Rice, J. B., \& Strassmeier, K. G. 1996, A\&A, 316, 164

Rice, J. B., \& Strassmeier, K. G. 2000, A\&AS, 147, 151 
Romanova, M. M., Ustyugovna, G. V., Koldoba, A. V., \& Lovelace, R. V. E. 2003, ApJ, 578, 420

Shu, F., Najita, J., Ostriker, E., et al. 1994, ApJ, 429, 781

Siess, L., Dufour, E., \& Forestini, M. 2000, A\&A, 358, 593

Slettebak, A. 1949, ApJ, 110, 498

Stempels, H. C., \& Piskunov, N. E. 2002, A\&A, 391, 595

Strassmeier, K. G., Pallavicini, R., Rice, J. B., Andersen, M. I., \& Zerbi, F. M. 2004, AN, 325, 278

Strassmeier, K. G., \& Rice, J. B. 1998, A\&A, 339, 497

Strassmeier, K. G., \& Rice, J. B. 2000, A\&A, 360, 1019

Strassmeier, K. G., \& Schordan, P. 2000, AN, 321, 277

Unruh, Y. C., Collier-Cameron, A., \& Guenther, E. 1998, MNRAS, 295, 781
Unruh, Y. C., Donati, J.-F., Oliveira, J. M., et al. 2004, MNRAS, 348, 1301

Walter, F. M. 1987, PASP, 99, 31

Wichmann, R., Bouvier, J., Allain, S., \& Krautter, J. 1998a, A\&A, 330,521

Wichmann, R., Bastian, U., Krautter J., Jankovics, I., \& Rucinski, S. M. 1998b, MNRAS, 301, L39

Wichmann, R., Krautter, J., Covino, E., et al. 1997, A\&A, 320, 185

Wichmann, R., Covino, E., Alcalá, J. M., et al. 1999, MNRAS, 307, 909

Zeipel von, H. 1946, ApJ, 103, 351 